\title{
¿Construcción con tierra en Puerto Rico? Conocimiento enterrado, sabiduría interrumpida
}

\author{
Earthen construction in Puerto Rico? \\ Buried knowledge, interrupted wisdom
}

Roselyn Méndez Resto*

Citar este artículo como: Méndez Resto, R. (2019). ¿Construcción con tierra en Puerto Rico?: Conocimiento enterrado, sabiduría interrumpida. Revista Nodo, 14(27), pp. 24-37

\section{Resumen}

En Puerto Rico existe evidencia de dos estructuras de tierra en el siglo XVI. La técnica que se utilizó fue la tapia. Este conocimiento no proliferó ni evolucionó. De hecho, hoy día no existen construcciones modernas hechas con tierra. La gente puertorriqueña promedio no conoce sobre las construcciones de tierra y debido a esa inexperiencia existe mucho escepticismo por la frecuencia de huracanes, la alta precipitación y la propensión a terremotos y maremotos. La pertinencia de encontrar precedentes históricos en Puerto Rico ayuda a entender el comportamiento de éstas a través del tiempo. Un sinnúmero de investigaciones apunta a que estas construcciones han sido efectivas en lugares con condiciones similares a las de la isla. En plena era del cambio climático es importante obtener conocimiento ancestral de materiales que han resuelto la necesidad de vivienda con un bajo impacto ambiental. Luego del paso del último huracán-María-, se enfrentó la realidad que existe cuando entendemos que la dependencia de la energía fósil y no renovable, la importación de los materiales de construcción por barco y el ejercicio de recuperarnos desde el gobierno (desde arriba), es muy frágil y susceptible. A través de una búsqueda de documentación en archivos históricos se pudo llegar a localizar y obtener información sobre la construcción con tierra. Según varias investigaciones científicas es posible enfrentar problemas ambientales a través de saberes que empoderan desde el pueblo y con ajustes en nuestro estilo de vida para entender así que las limitaciones se encuentran en el desconocimiento.

Palabras clave: tapia, Caribe, trópico, dependencia, autogestión, regeneración.

\section{Abstract}

In Puerto Rico, there is evidence of two earth constructions for the 16th century. The technique used was rammed earth. This knowledge did not proliferate or evolve, in fact, today there are no modern constructions made on earth. The average of Puerto Rican does not know about earth constructions and due to this inexperience there is much skepticism about the frequency of hurricanes, high precipitation and being prone to earthquakes / tsunamis. The relevance of finding historical precedents in Puerto Rico would help

Fecha de recepción: 26 de julio de 2019 - Fecha de aceptación: 26 de septiembre de 2019

* Arquitecta, Universidad de Puerto Rico. Correo electrónico: roselyn.mendez@gmail.com 
to understand their behavior over time. Countless research indicates that these constructions have been effective in places with similar conditions those the island. In the era of climate change, it is important to obtain this ancestral knowledge of materials that have solved the need for housing with a low environmental impact. After the passage of the last hurricane Maria, Puerto Rico faced the reality that exists when we understand the dependence on fossil and non-renewable energy, the importation by ship of building materials and the exercise of recovering from the government (top to bottom), It is very fragile and susceptible. Through a search of the documentation in Historical Archives, it was possible to locate and obtain information on the earth construction. According to several sources of scientific research, it is possible to face environmental problems based on the knowledge that empowers from the town and with adjustments in our lifestyle, it was understood that the limitations are in the ignorance.

Keywords: rammed earth, Caribbean, hurricane, earthquakes, Puerto Rico, tropics.

\section{Introducción}

Los materiales de construcción que se utilizan en la isla tropical de Puerto Rico son la madera, el cemento y el acero (material necesario para las construcciones en cemento). Casi no existe otra opción. Pero, ¿por qué? ¿Será que son las opciones, económicamente, más accesibles? En términos generales, la selección del material no tiene que ver con los niveles de pobreza, pues hacer una construcción en madera en Puerto Rico, no es económico. La madera que se consigue en el mercado no es local y tampoco la de mejor calidad. Esto incrementa el precio y, a la vez, la huella ecológica. Además, son muy pocos los aserraderos de madera local y es un trabajo artesanal muy valioso que no todos ni todas podemos costear. El arquitecto Fernando Abruña (2010), considerado como el padre de la arquitectura verde en Puerto Rico afirma:
Toda la madera que utilizamos en Puerto Rico es importada del exterior (...). Con frecuencia no sabemos la procedencia de ésta. Pensando que la madera es un material restituible en la naturaleza podríamos estar patrocinando, sin saberlo, algún aserradero que opera sin las mínimas estructuras operacionales, haciendo talas indiscriminadas de árboles creando las condiciones que abonan a la desertificación de los bosques. Si a esto añadimos que las maderas que utilizamos en su gran mayoría están protegidas de la polilla y el comején con químicos tóxicos al ambiente y al ser humano, el escenario se complica aún más. Otra consideración al hacer uso de la madera como material de construcción tiene que ver con la rapidez en que la Naturaleza se re-establece del daño que se le hace al talar un árbol para uso en la construcción (...) la mera decisión del tipo de madera que utilizamos hace una gran diferencia en el tiempo que le toma a la Naturaleza "curar su herida" (p. 20) ${ }^{1}$.

Según la arquitecta especializada en conservación histórica, Beatriz Del Cueto (2014), "algunas de las tecnologías tradicionales de construcción originales a nuestra región antillana (madera, bahareque, tapia, mampostería y cantería) ya no se utilizan y la industria de la construcción contemporánea, en gran medida, ha olvidado las mismas" (p. 16). También expone que, según los trabajos de inspección realizados tras los terremotos de 1918, los edificios de madera "subsistieron excepcionalmente bien” (p. 17). La selección y uso de los materiales actuales son el resultado del desconocimiento de otras opciones posibles, de las regulaciones del mercado y del sistema, sumado a la seguridad aparente que nos ha brindado las estructuras de cemento. Sin embargo, existe conocimiento que, en el caso de terremoto, la flexibilidad de la madera tiene un punto a su favor; todo depende de muchos factores que hay que tomar en consideración.

El cemento tiene el beneficio de demostrar resistencia a huracanes y terremotos, de ser

1 Es el fundador y ex presidente del capítulo del Caribe del Green Building Council. 
impermeable y manufacturarse localmente. Sin embargo, depende de una estructura de acero que debe ser importada y que es vulnerable al salitre, mayormente en las zonas costeras. Que el cemento sea producido localmente no significa que sea un producto amigable con el ambiente, ya que la extracción del material entraña minar los mogotes de la isla, su producción implica el uso de la energía eléctrica y del petróleo, emana particulado y liberación de $\mathrm{CO} 2$ y es un material que difícilmente se puede volver a reincorporar a la tierra pues es un producto manufacturado y procesado. En el mundo, la industria insostenible de la construcción consume más recursos que cualquier otra actividad económica $^{2}$ (Pacheco-Torgal \& Jalali, 2011). Al combinarse con la carencia de conocimiento y de construcciones bioclimáticas adaptadas al trópico, nos vemos en la obligación de utilizar tecnologías de enfriamiento artificiales como acondicionadores de aire que pueden provocar crecimientos de hongo y crear edificios enfermos que contagian al que los habita. Es importante recalcar que, en Puerto Rico, existe una asociación entre seguridad y el cemento debido al resultado experimentado por la población luego de un huracán, como se evidenció recientemente (septiembre de 2017) cuando se vivió el paso de María con categoría $4^{3}$, donde la mayoría de las viviendas destruidas fueron las de madera y no las de cemento. ${ }^{4}$ Además de los huracanes, ${ }^{5}$ Puerto

\footnotetext{
2 "About $3000 \mathrm{Mt} /$ year, almost $50 \%$ by weight" (PachecoTorgal \& Jalali, 2011).

3 Se cree que en algunas zonas se sintió de categoría 4 y
} en otras de 5 , dependiendo de la posición del lugar respecto al paso del ojo y la altura.

$4 \quad$ Una vivienda de cemento puede presentar daños dependiendo de su ubicación, pero no es la norma. Según la Central Meteorológica y Geológica del Caribe un techo de cemento fue "levantado y destruido parcialmente" por el huracán María el 20 de septiembre de 2017. Esta residencia se encuentra en el municipio de Yabucoa, situado en la costa sureste de Puerto Rico, y uno de los primeros lugares en recibir las ráfagas del huracán. En la foto de la residencia, se pueden observar las varillas completamente dobladas hacia la dirección que el viento las azotó.

5 Puerto Rico ha recibido más de 15 huracanes reportados desde el 1508. El primero lo denominaron la Tormenta
Rico también ha experimentado terremotos al estar ubicada sobre la placa tectónica del Caribe. El último de gran intensidad que presenció la isla (7.3 en la escala Ritcher), fue el del 11 de octubre de 1918 (Red Sísmica, 2019), seguido de un maremoto.

El hecho de que no exista casi ninguna otra opción, entre los materiales de construcción, está vinculado a la inaccesibilidad de información sobre otras opciones posibles como lo es la construcción con tierra. No existe una transmisión de conocimiento heredado porque se rompió ese eslabón. Solo tenemos constancia de un pedazo de edificio erigido en tapia y testigo presencial de la mayoría de los huracanes y terremotos que han sido reportados en la historia de Puerto Rico. Nuestra posición geográfica solo nos pone otros retos estructurales que ya han sido resueltos en otros países y que son parte de otros pasos a dar en esta investigación, pero el material y la necesidad son reales. La dependencia, importación y vulnerabilidad que representa la obtención de nuestros materiales, en plena era del cambio climático y siendo una isla colonial, son los cimientos para reconocer que se deben abrir las puertas hacia otras formas y maneras posible de construir.

San Roque (16 de agosto de 1508). Esta fue la época en que los españoles intentaban establecerse en el país y los reportes que existen del mismo vienen directamente desde España. Luego, varios años después llega el primer huracán oficialmente reportado llamado San Felipe II (año 1928). Este huracán fue categoría 5 y causó muchas muertes y pérdidas materiales. Cuatro años después la isla fue afectada por el huracán San Ciprián (26 de septiembre de 1932) y no fue hasta 24 años después que Puerto Rico se sorprende con la llegada de Santa Clara (11 de agosto de 1956). En los años 70, fueron reportados Eloísa (15-16 de septiembre de 1975) y David (30 de agosto de 1979) pero fue con la llegada del huracán Hugo (18 de septiembre de 1989) que la generación X experimentó lo que significaba un huracán categoría 3 por primera vez. Luego, casi con la misma intensidad, vivimos Marilyn (16 de septiembre de 1995), Hortense (9-19 de septiembre de 1996), Georges (21 de septiembre de 1998) e Irene (21-22 de agosto de 2011 (Metro, 2017). Recientemente sufrimos la amenaza de dos huracanes categoría 5 y con solo días de diferencias Irma y María. El huracán Irma hizo mucho daño a la vegetación amortiguadora hasta llegado el huracán María, en un inolvidable 20 de septiembre de 2017. 


\section{Evidencia de construcción con tierra en Puerto Rico}

Para encontrar esas primeras construcciones de tierra se realizó una búsqueda en el Archivo Histórico de Puerto Rico y en la Colección Puertorriqueña de la Universidad de Puerto Rico, en el Museo Ruinas de Caparra y Museo Casa Blanca de Juan Ponce de León. Reconociendo el valor y experiencia de dos expertos en sus áreas profesionales, debo recalcar que se acudió a la arquitecta de Conservación, Beatriz del Cueto y al historiador Johnny Lugo Vega para que sirvieran de guías en el proceso, lograr esclarecer dudas y poder llegar a los recursos y fuentes de información de manera más clara y precisa; de esta manera se pudieron obtener los datos. Luego se procesó la información y se realizó una revisión de literatura que respaldara la posibilidad de este tipo de construcción en nuestra isla. Obtener información sobre estas estructuras nos ayudará a esclarecer preguntas sobre cómo se han comportado y adaptado al trópico húmedo, caribeño e insular.

Solo hay certeza de dos ejemplos de construcción con tierra, hechas con la técnica de tapia. La evidencia encontrada señala que la primera construcción de tierra en Puerto Rico fue en 1508 y corresponde a la casa de Juan Ponce de León, erigida para defenderse de los indios taínos, ya que éstos quemaban las estructuras de madera y todo intento de establecimiento invasor por parte de los españoles. Este prototipo solo existe en fotos que se encuentran en el Archivo Histórico de Puerto Rico y en la Colección Puertorriqueña de la Universidad de Puerto Rico gracias al trabajo realizado en 1938 por el historiador Adolfo de Hostos. Dicha vivienda se abandonó y quedó en el olvido. Su descubrimiento oficial implicó exhumar, literalmente, de una montaña de tierra, escombros y vegetación que prolifera y recubre todo en el trópico. En 1938 se desenterró esta vivienda y con ella esa primera evidencia de este tipo de construcción. Lo que observamos hoy día (Figura 1) no es igual a lo que podemos contemplar en las fotos
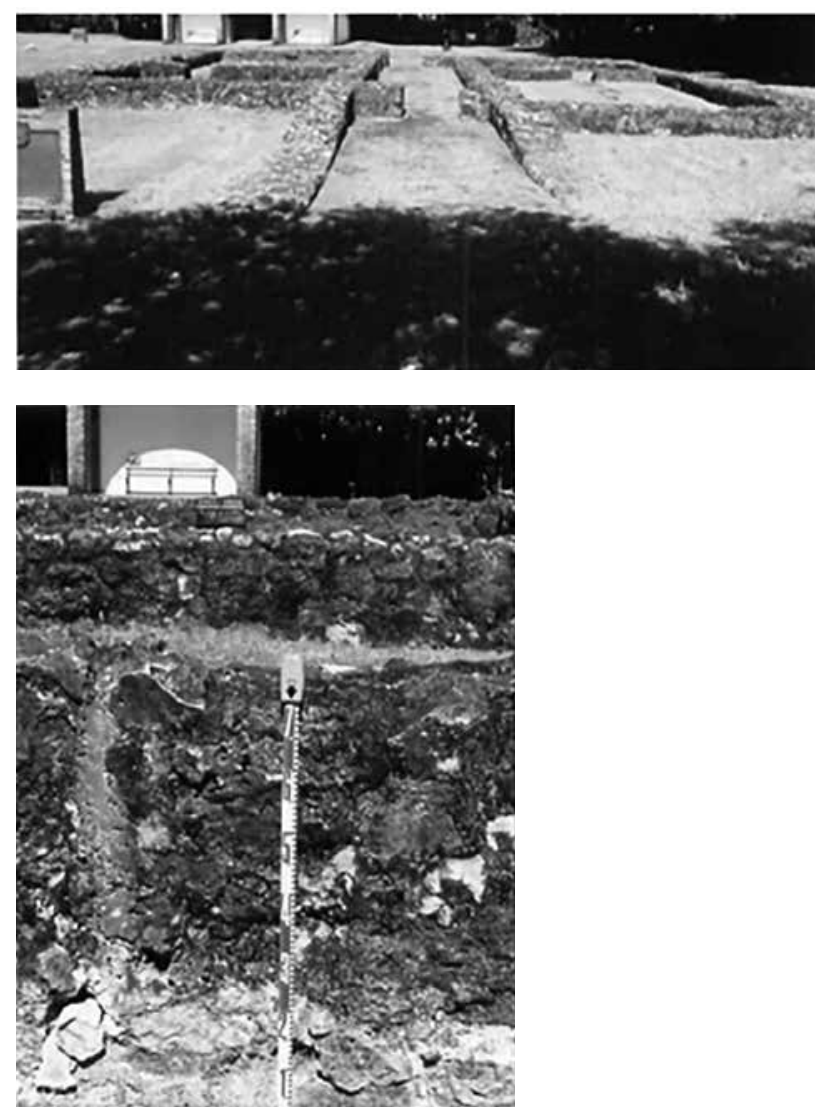

Figura 1. Condición actual de las ruinas de Caparra Fuente: Pantel et al. (1988).

del trabajo de Adolfo de Hostos (Figura 2). En las imágenes de Hostos, podemos percibir parte de las paredes de tierra. Lo que hoy llamamos ruinas y podemos visitar es una réplica moderna cuya ubicación tampoco corresponde a la que existió en aquel momento (Figura 3).

Esta evidencia se encuentra en el hallazgo arqueológico contenido en Archeological, Architectural and Historical Investigations of the First Spanish Settlement in Puerto Rico (Pantel et al., 1988), donde se efectúa una comparación de fotografías aéreas del año 1937 y se aprecia que la disposición de la verdadera ruina se encontraba en diagonal con la carretera llamada Militar y que coincide con la documentación suministrada por Adolfo de Hostos (Figuras 4 y 5 ). Lo que podemos deducir de la investigación, es que la Carretera Militar (conocida también como la carretera número 

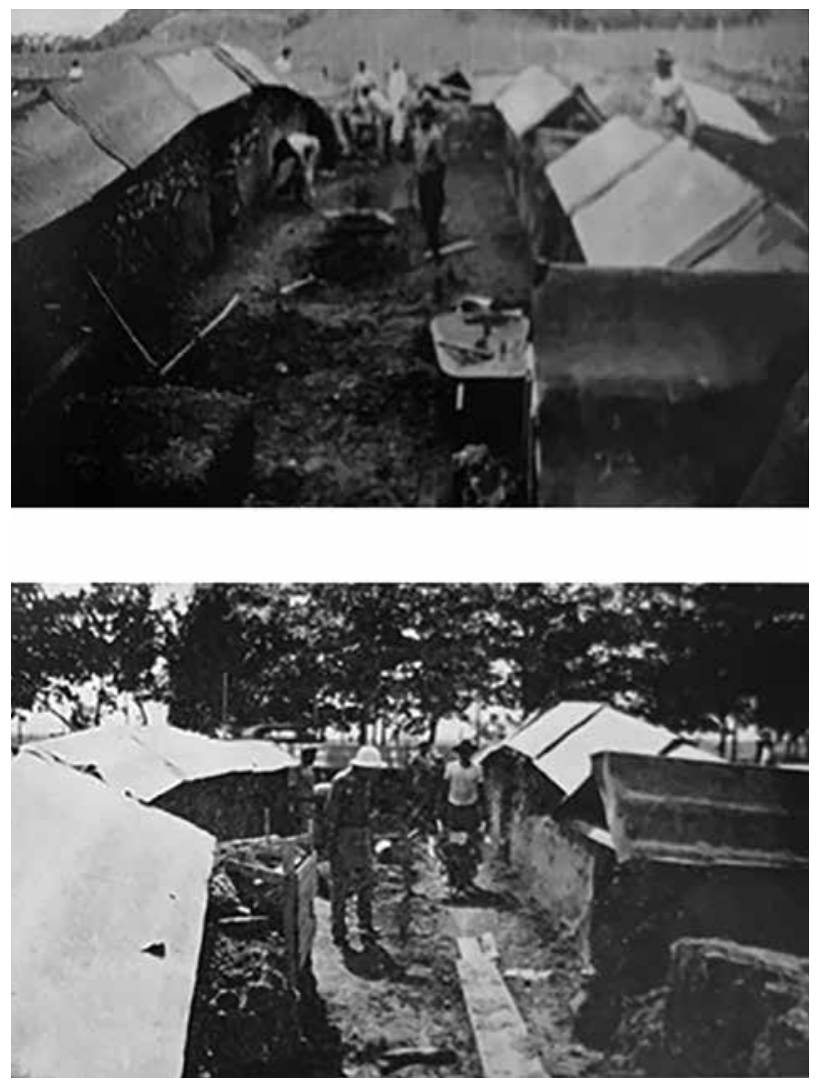

Figura 2. Excavación de la vivienda de Juan Ponce de León en Caparra. Evidencia de paredes de tapia. Fuente: De Hostos (1938).

dos) atravesó completamente y dividió en dos mitades lo que fue esa primera residencia. Hoy día, se observa una estructura formando la huella de la planta en piedra y con aparente mortero de cemento en una disposición perpendicular, en vez de diagonal como la original, tal y como lo muestra la Figura 3 (Pantel et al., 1988, p. E-5).

Sin información original sobre esta estructura, es difícil llegar a conclusiones pues no podemos asumir cómo se ha comportado en el tiempo. Solo podemos suponer que su exhumación la perjudicó, pues una construcción con tierra debe ser protegida de las inclemencias del tiempo. Aun así, las condiciones en las que se encontraba, antes de ser descubierta, eran de abandono y deterioro. El valor de la documentación en fotos y los trabajos de Adolfo de Hostos permiten afirmar que sí era una construcción en tapia y que con tal material se
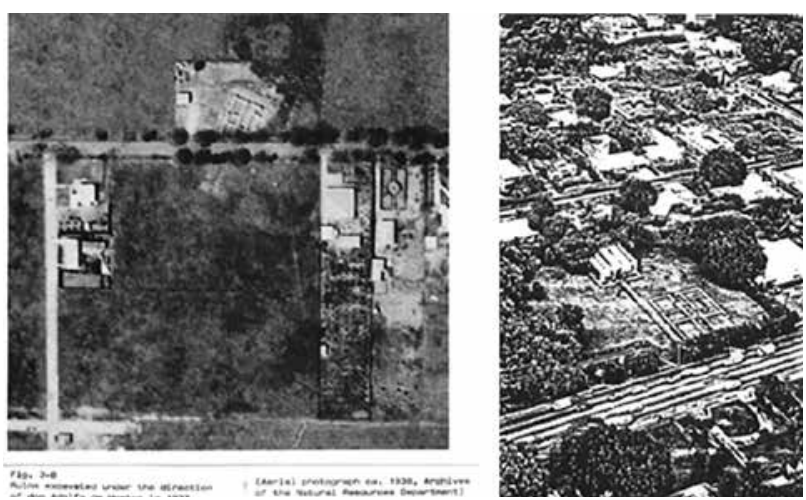

Figura 3. A la izquierda, foto aérea de 1937 con una disposición en diagonal a la carretera; a la derecha, foto de 1979 con estructura en una disposición perpendicular a la carretera Fuente: De Hostos (1938); Pantel et al. (1979).

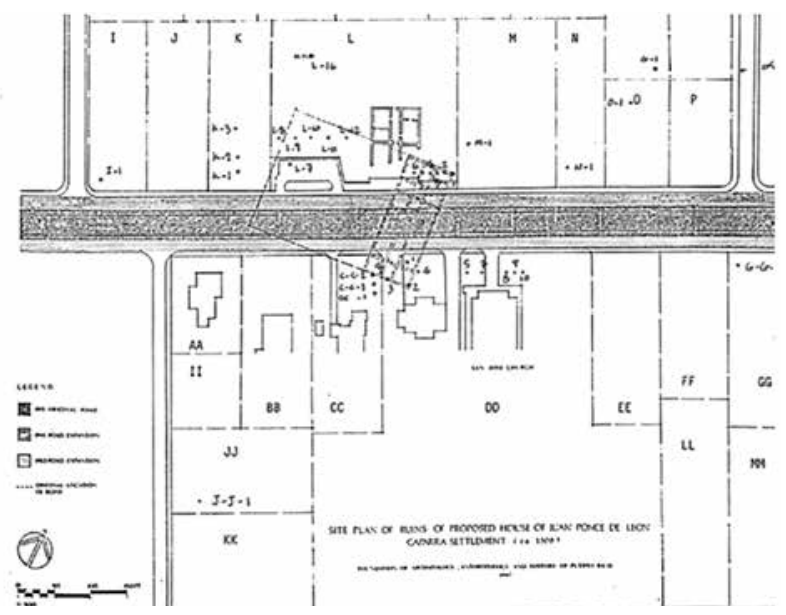

Figura 4: Trabajo arqueológico con ambas estructuras superpuestas. Fuente: Pantel et al. (1988).

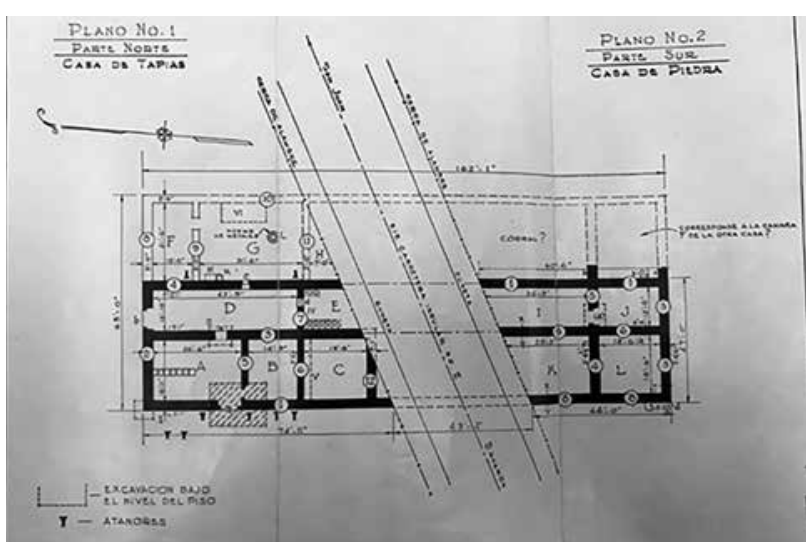

Figura 5. Plano de lo que era la residencia de Juan Ponce de León en Caparra con la carretera atravesándola de forma diagonal. Fuente: De Hostos (1938). 
logró el establecimiento de la primera residencia permanente de Juan Ponce de León.

Otra construcción con tierra fue la segunda residencia de Juan Ponce de León. Hoy día esta edificación es custodiada por el Instituto de Cultura Puertorriqueña y es declarada por la Asamblea Legislativa, como Monumento Histórico Nacional. En los trabajos de Hostos se hace mención que la razón de mudarse a la isleta del Viejo San Juan era por seguridad y por la molestia que se producía al vivir en una zona muy propensa a mosquitos (De Hostos, 1938). Pero nunca se menciona que la razón de mudanza estaba relacionada con algún problema de la vivienda. Esta nueva residencia es hoy día el museo Casa Blanca (Figura 6). Es un museo temático sobre la familia de Juan Ponce de León. Esta estructura se realizó en fases y la primera de ellas fue hecha en tapia en el año 1521-1523. Constó solo de una habitación de 24 pies cuadrados. En una visita al museo pueden contemplarse estas paredes que están expuestas al público en general (Figura 7).

Este segmento de estructura de tapia se encuentra en buen estado y en uso a pesar de sus 498 años en pie. No es un cuarto interior, sino que forma parte de la esquina de la residencia que da a la intemperie, como se puede observar en la zona marcada de la Figura 7. Este sería el único segmento de edificio constituido y en funcionamiento de tapia que existe en Puerto Rico. Ha experimentado la mayoría de los huracanes y terremotos que han sido reportados en la historia de nuestra isla. Se erigió en 1521 y presenció alrededor de 11 huracanes ${ }^{6}$. Aunque se han reportado varios terremotos, uno de los más destacados de mencionar fue el ocurrido en el año 1787 con un magnitud de 8.0. Ocurrió en la Trinchera de Puerto Rico y se reportaron daños considerables en la isleta de San Juan, donde precisamente se ubica Casa Blanca. Es interesante recalcar que evidentemente la

6 El huracán San Felipe del año 1928 de categoría 5, San Ciprian (1932), Santa Clara (1956), Eloísa (1975), David (1979), Hugo (1989), Marilyn (1995), Hortense (1996), Georges (1998), Irene (2011) y María (2017).

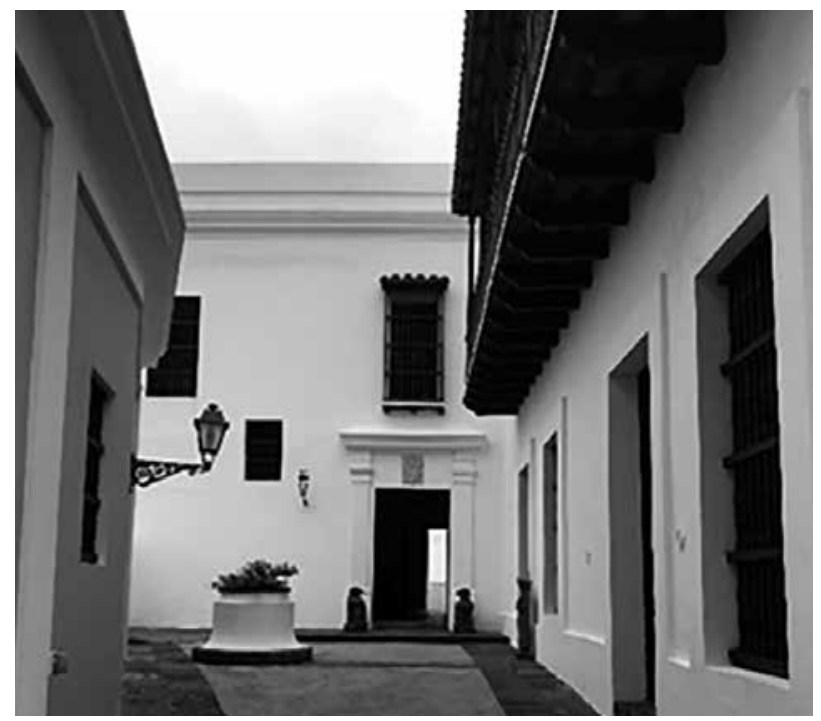

Figura 6. Condición actual del Museo Casa Blanca. Fuente: elaboración propia.

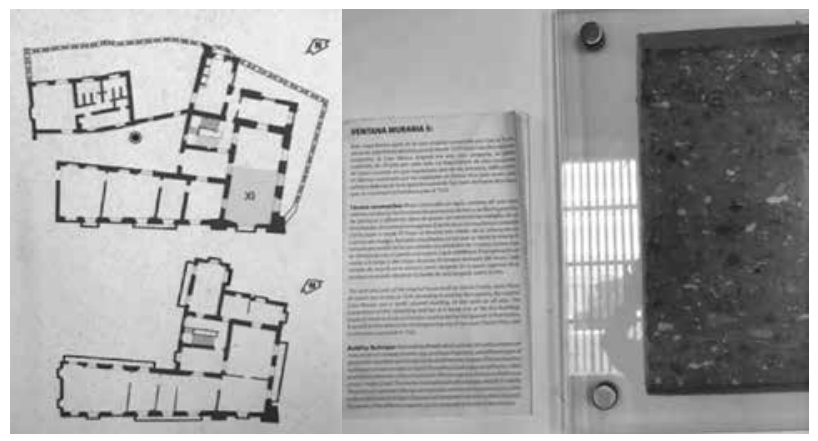

Figura 7. A la izquierda se observa resaltada la parte construida en tapia; a la derecha, un pedazo expuesto de la pared de tapia. Fuente: Museo Casa Blanca de Juan Ponce de León en el Viejo San Juan de Puerto Rico.

estructura completa lo superó, mientras que según informes del Cabildo de San Juan en 1787, hubo paredes de los castillos San Felipe del Morro y San Cristóbal que presentaron mucho daño estructural y grietas en los muros (Ecoexploratorio, 2018).

Las nuevas expansiones de la residencia se comenzaron a hacer con otras técnicas constructivas que luego se mantuvieron constantes en todo Puerto $\mathrm{Rico}^{7}$, tales como la mampostería (mampuestos

7 Hay otros lugares en los que se cree existen construcciones de tapia pero, por el momento, no pudimos obtener las respectivas evidencias. Se cree que el Castillo Rojo y la 
de roca y ladrillo cocido exportado de España) con morteros hidráulicos de cal y ladrillos o teja molida. Según Beatriz Del Cueto (2013):

En Puerto Rico, los morteros hidráulicos han sido utilizados para construir desde el siglo XVI, cuando, como colonia española, se heredaron las tradiciones edilicias ibéricas. Fue posible erigir estructuras militares, religiosas y civiles permanentes con el uso de cal y aditivos hidráulicos como ladrillo o teja molida. Estos edificios de albañilería probaron ser resistentes a las tormentas tropicales y al clima marítimo de la zona (p. 12).

Esta fue la técnica constructiva que, en algún momento de la historia, predominó en el país. Aún hoy las podemos encontrar en los centros urbanos (históricos) de casi todo Puerto Rico. Con el problema que su reconstrucción, restauración y/o conservación, muy pocas veces se encuentra en manos expertas. El mayor y clásico error, como ocurre en otros países, es utilizar un material no compatible para el revoque o restauración. En nuestro caso se utiliza cemento, pues:

Durante el siglo XIX y principios del siglo XX, las tradiciones constructivas en la Isla evolucionaron grandemente con el uso del polvo de ladrillo o cemento artificial, materiales necesarios para preparar mezclas hidráulicas. El cemento Portland genuino fue importado a la Isla desde la década del 1850 procedente de Bélgica, Alemania y Dinamarca (Del Cueto, 2013, p. 12).

En el contexto histórico de ese entonces, se debe aclarar que en 1898 acontece la segunda invasión por parte de Estados Unidos a Puerto Rico:

Como consecuencia de la Guerra Hispanoamericana, el nuevo Gobierno insular emprendió un ambicioso plan de construcción de carreteras, puentes y escuelas. Gran cantidad de los edificios institucionales y de vivienda de la época utilizaron cemento Portland como parte de la mezcla para el hormigón armado, imitando

Torre La Fuerza también son en tapia. Investigaciones futuras podrían confirmarlo. modelos introducidos por el Gobierno norteamericano, grupos religiosos protestantes y los militares (Del Cueto, 2013, p. 13).

Precisamente, la carretera "La Militar" fue una de esas construcciones que atravesó la antes mencionada primera edificación de tapia y piedra en Caparra.

\section{¿Se puede hacer construcción con tierra en Puerto Rico?}

A continuación se enumeran algunas fuentes que esbozan ventajas y desventajas, con diferentes condicionantes, a tener en cuenta a la hora de hacer construcción con tierra en Puerto Rico.

Según Rempel \& Rempel (2016), las propiedades higroscópicas de las paredes de tierra son capaces de regular la temperatura logrando un confort interior (p. 1). Por lo tanto, en una isla caribeña de clima húmedo tropical, la construcción con tierra podría resultar muy beneficiosa pues interactúa con el medio ambiente logrando estabilizar a temperatura agradable. En un estudio de Suryandari y Mubin (2007), realizado sobre unión de tecnologías modernas y tradicionales para arquitectura sostenible de viviendas en el trópico, se reconoce que muchas edificaciones tradicionales no son costosas y son amigables con el ambiente; el problema es que no han sido capaces de resistir desastres naturales. Dicho escrito trata de la región de Indonesia, que se ha caracterizado por experimentar terremotos, erupciones de volcanes, inundaciones, movimiento de masa, sequías e incendios forestales. La vulnerabilidad a terremotos y huracanes hace dudar de la tierra como material de construcción. La alta precipitación de la isla recuerda constantemente que estas estructuras deben estar protegidas de los disturbios tropicales (escorrentía, inundaciones, fallas de taludes, etc.). Los mecanismos de destrucción por huracanes son, según Houben y Guillaud (2014), la erosión del suelo, la presión del aire, la erosión en cimientos, la succión de la pared y la succión del techo. 
Según un análisis patológico de los daños típicos estructurales causados por los ciclones, no existe una estructura estándar sismo resistente por lo que se considera la estabilidad de la edificación en su conjunto y la resistencia al viento de cada elemento: "no lateral force should be able to move or turn the building over" (p. 322).

Un factor muy importante para tener en cuenta es el buen o mal manejo de selección de material, mantenimiento y construcción. La arquitectura con tierra ha sido estigmatizada porque hay ejemplos donde la estructura ha fallado por fuerzas telúricas, porque se ha deteriorado rápidamente por el agua o ha sido invadida por agentes biológicos como hongos, termitas, el mal de chagas y hasta por orificios creados por roedores. Pacheco-Torgal \& Jalali (2011) consideran que son materiales menos duraderos comparados con materiales convencionales, "less durable as a construction material compared to conventional materials" (p. 515); sin embargo, en el mismo escrito, los autores aceptan que esto ocurre por malas decisiones o desconocimiento de información a la hora de efectuar estas construcciones. La presencia de insectos y plagas dentro de los muros de tierra merecen preocupación, pero este aspecto es solo un problema de descuido de los muros al sufrir erosión severa ${ }^{8}$. Esta misma investigación argumenta que las construcciones con tierra han sido capaces de durar miles de años.

Otras contradicciones que podemos observar en el estudio de Pacheco y Jalali son referentes a que la construcción con tierra, no son posibles en zonas sísmicas sin estructuras de hormigón armado, lo que reduce su ecoeficiencia. Esta afirmación se basa en dos investigaciones que utilizan solo las técnicas de tapia y adobe y con un solo tipo de diseño de planta cuadrada. En contraposición, es importante recalcar lo que Houben y Guillaud (2014) exponen cuando se refieren a que la patología reflejada en edificios de tierra que son afectados por terremotos,

8 "Other authors recommend the use of bactericidal mixtures to prevent this problem". son el resultado de una construcción descuidada y mantenimiento defectuoso:

The weakness of earth as a building material is
increased by ignorance and lack of supervision at
all stages of the production of the material, of the
design and of the construction of buildings. Earth
structures, like other masonry structures, do not
have much resistance to stresses of seismic origins.
The poor bond between its various elements and
the weakness of the bearing walls are severely
punished (p. 312). Por lo tanto, podemos afirmar que el material (tierra, madera, cemento o acero) no son los determinantes. Lo son las malas decisiones que se toman antes, durante de la construcción y en el uso de la edificación.

Pacheco-Torgal y Jalali también mencionan que las casas de tierra poseen un mal comportamiento en los casos de terremoto (2011, p. 515). Pero toda construcción debe tener en cuenta el lugar donde será realizada y que el nivel de daño está íntimamente relacionado con la naturaleza geológica y topográfica del lugar (Houben y Guillaud, 2014). Cualquier edificación que no tenga un buen mantenimiento, utilice materiales de mala calidad, esté estructuralmente mal construida y en zonas de alto riesgo, será de corta duración y representa un peligro, en términos estructurales y para quien la habite.

La construcción con tierra es susceptible al agua y por lo tanto hay que trabajarla con esta consciencia. Un lema popular que se escucha entre bioconstructores es que "la construcción con tierra debe tener buenas botas y buen sombrero". Es decir, que debe tener un cimiento y sobrecimiento impermeable y voladizos/aleros que protejan la estructura de la gota en diagonal y constante, de la erosión por salpicadura y la erosión por capilaridad. El principal responsable de la erosión de las paredes tiene que ver con la energía cinética del impacto de la lluvia (Pacheco-Torgal \& Jalali, 2011). Por lo que, en un país donde no se utiliza la tierra para la construcción, se sugiere que la introducción de ese saber ancestral, ocurra en transición y poco a poco. 
En los inicios podemos aprovechar dos materiales de construcción (cemento impermeable) para ese sombrero $^{9}$ y botas, pero vestimos y abrigamos la edificación con la tierra, como material que interacciona con el ambiente del trópico. En Estados Unidos existe la regulación E2392/E2392M-10 Standarg Guide for Design of Earthen Wall Building Systems, que aplicaría en Puerto Rico. Las construcciones con tierra hechas por vecinos caribeños, como lo expone Esteban Prieto Vicioso (2015), nos hablan de una construcción adaptada a nuestro clima como en la República Dominicana. Por tal razón se recomienda ampliar el estudio al Caribe antillano que comparte nuestra misma geografía y parte de nuestra historia.

La búsqueda de diferentes técnicas de construcción con tierra en el Caribe nos puede ofrecer una respuesta de cómo estos materiales se adaptan al trópico de Puerto Rico. El conocimiento de otros materiales más accesibles y con características sostenibles resulta imperativo teniendo en cuenta el efecto del cambio climático. La construcción con tierra acorta la huella ecológica y tiene la capacidad de traer confort térmico interior sin la necesidad de añadir tecnologías de enfriamiento artificial en un clima húmedo tropical donde el calor fluctúa entre $68^{\circ} \mathrm{F}$ y $86^{\circ} \mathrm{F}^{10}{ }^{10}$ Además, un estudio en Nigeria de Onyegiri y Ugochukwu (2016) demostró las ventajas de utilizar materiales tradicionales de construcción (adobe, bambú, madera y otras) por su disponibilidad y asequibilidad y porque son materiales se biodegradan (p. 247).

\section{Conocimiento enterrado, sabiduría interrumpida}

El material (tierra, madera, cemento o acero) no son los determinantes de riesgo, sino las malas

\footnotetext{
9 Sombrero alusivo al alero más no a los techos, éstos pueden ser en bóvedas o cúpulas con bloques de tierra comprimida (BTC) o en madera con techo verde. Los techos se deben trabajar como estructuras independientes a los aleros porque si el viento vuela los aleros no debería afectar los techos.
}

10 Según el libro Tropical Geomorohology (Gupta, 2011). decisiones que se toman antes, durante de la construcción y en el uso de la edificación. El saber y la transferencia de conocimiento empodera comunidades que, precisamente, han sido desprovistas de tal sabiduría ancestral. De esta manera podemos asociar dicho conocimiento al concepto que implica el llamado "decrecimiento sereno" de Serge Latouche que implica reducir o suprimir las cargas al ambiente (2003, p. 3). De aquí la interrogante sobre la importación y exportación de otros materiales y también el argumento sobre la durabilidad que puede representar una edificación de tierra, bien construida y con buen mantenimiento. Porque pequeñas intervenciones para la introducción del material de tierra implican reducir el uso del cemento y la carga al ambiente que este genera.

Uno de los condicionantes del ecofeminismo constructivista propuestos por Yayo Herrero (2006) es el "inevitable decrecimiento de la esfera material de la economía" (p. 25) donde por las buenas se decrecerá de forma planificada y justa ya que por las malas más personas no pueden acceder a los mínimos materiales de existencia digna.

En Puerto Rico, los materiales que usamos para construir son inaccesibles por ser manufacturados e importados y por depender de mano de obra especializada. Esto hay que tenerlo en cuenta, en caso de crisis. Tampoco existe el conocimiento de lo que es la construcción con tierra. Así que ni por materiales ni por transmisión de conocimiento podemos acceder a los "mínimos materiales de existencia digna". No solo tenemos miopía hacia un recurso, sino que se nos ha quitado el acceso a esa información que ha permitido a miles de personas, en el mundo entero, tener una vivienda para refugiarse.

Dado que en Puerto Rico no existe la cultura de hacer construcción con tierra podemos comenzar el diálogo desde el conocimiento adquirido en otros países tanto en la parte académica como en la popular. En el prólogo del libro Test Carazas Manual Pedagógico, la arquitecta Sandy Minier menciona que "la devolución de conocimientos con respaldo científico valora los saberes ances- 
trales, reconstruye la autoestima y la autonomía" (Carazas, 2017, p. 3). Sabemos que es una arquitectura que necesita mantenimiento, estar estructuralmente bien construida y la selección del material es clave para que sea una construcción duradera y sana. Carazas indica que: "(...) aquel que conoce mejor la tierra tendrá mejores argumentos constructivos para utilizarla, conocerla bien para después proponer otras alternativas" (p. 1).

Una vez introducido el conocimiento de cómo se hace construcción con tierra en Puerto Rico y de tener las primeras estructuras que nos sirvan de precedente, la evolución y las tecnologías criollas proliferarán. Puerto Rico posee 10 de los 12 órdenes de suelo reconocidos por "Soil Taxonomy", a pesar de ser un espacio relativamente pequeño (111 x 39 millas cuadradas) si lo comparamos con otras partes del mundo que pueden tener mayor extensión territorial pero muy poca variedad de suelos. No existen Andisoles (porque ya no hay actividad volcánica) ni Gelisoles (por ser trópico húmedo, y no cae nieve). Esta diversidad de suelos abre toda una gama de posibles investigaciones, con sus implicaciones geográficas:

The tropical climate of the Island precludes the formation of Gelisols, soils that must have permafrost. The other order not present is Andisols because Puerto Rico, although largely of volcanic origin, lacks the recent volcanic materials that are a prerequisite for their formation since active volcanism ceased about 70 million years ago (Muñoz et al., 2016, p. 4).

\section{Conocimiento enterrado: semilla que germina en el futuro}

En plena era del cambio climático es importante hablar sobre temas y propuestas para lograr transformaciones en los estilos de vida, en las decisiones que tomamos diariamente y que afectan al ambiente. Es momento de abrirnos a las posibilidades ancestrales, aquellas a las que el tiempo les ha dado la razón. Ted Trainer (2014) lo expone de la siguiente manera:
The best way to get a house is not by ordering one to be laser-printed but by enjoying slowly building it by hand with friends, from mud. We have known for centuries how to make perfectly satisfactory housing, food, clothing, pottery, music, community, conversation and entertainment. (p. 173)

Nos hemos desconectado de los procesos de la manufactura de un producto. De este modo, no somos capaces de entender si los procesos son lineales o de manera circular. Además, perdemos de perspectiva el valor que tiene el producto final de la comida, de la vestimenta, de cualquier objeto y de nuestra propia vivienda.

Con el más reciente paso del huracán María hemos experimentado, a modo de simulacro, lo que es el colapso de la infraestructura de comunicaciones, electricidad, agua potable y de algunas viviendas; la falta de alimento y la vulnerabilidad de los hospitales y el sistema forense. También de la fragilidad de los medios de importación, a través de embarcación, donde llegan los materiales de construcción y el $80 \%$ de nuestra comida y materiales. Mientras que por parte del gobierno, la estrategia de la supuesta recuperación parecía estar más sumergida en lo que Naomi Klein (2017) llama el "capitalismo del desastre" y expone que los "acontecimientos de carácter catastrófico", son simultáneamente atractivas oportunidades del mercado (p. 26), muchas veces con intereses de carácter individual y no en función del colectivo. Sin embargo, pudo observarse que inmediatamente después del huracán el pueblo se organizó, de manera orgánica y desde abajo, para limpiar sus calles de los escombros, estableciendo comedores sociales en comunidades, haciendo huertos comunitarios, estaciones para filtrar el agua y potabilizarla, contribuyendo en la reconstrucción, entre otros. Se comenzó el diálogo sobre las comunidades solares, se recogieron los árboles caídos para aserrarlos y darles uso. Todo esto bajo la impotencia de observar cómo con los mismos materiales naturales que había que remover de las calles - madera y la tierra de los deslizamientosse podía rehacer tantas viviendas que ahora ya no existen. Yayo Herrero (2016) explica que: 
Estas experiencias son laboratorios sociales, a la vez que satisfacen las necesidades concretas de quienes participan en ellas. Alentar estos proyectos, aunque sean pequeños y no supongan una alternativa global, es muy importante porque además preparan a las personas y colectivos para disputar el poder en todos los ámbitos. La participación del ecologismo social en todas estas iniciativas es intensa y constituye también una importante línea de trabajo (p. 28).

Puerto Rico está listo para comenzar el diálogo sobre nuevas alternativas y diferentes estilos de vida que prometan regenerar un país que sobrevivió a un huracán como el María. Luego del trauma posthuracán se ha observado claramente la "táctica del capitalismo contemporáneo" que Klein (2017) denomina "doctrina del shock" y donde "la crisis (...) da lugar a un cambio verdadero" (p. 27), con posturas o visiones distintas del gobierno (desde arriba) comparadas con las necesidades reales del pueblo (desde abajo).

Max-Neef, Elizalde y Hopenhayn (2010) proponen un "desarrollo a escala humana" sustentado en la satisfacción de las necesidades humanas fundamentales a través de la autodependencia y la articulación orgánica entre los seres humanos construida a partir del protagonismo real de las personas privilegiando la diversidad y autonomía de los espacios. De esta manera:

Lograr la transformación de las personas-objeto en persona-sujeto del desarrollo es, entre otras cosas, un problema de escala; porque no hay protagonismo posible en sistemas gigantescos organizados jerárquicamente desde arriba hacia abajo (...) apunta hacia una necesaria profundización democrática. Al facilitar una práctica democrática más directa y participativa, puede contribuir a revertir el rol tradicionalmente semi-paternalista del Estado latinoamericano, en rol estimulador de soluciones creativas que emanen desde abajo hacia arriba ${ }^{11}$ y resulten,

11 Mayerfeld (2012) llama a esto "ecological dialogue: Dialogue of bottom and top (...) a bottom-up community may have a strong dialogue of solidarities going, and thus a strong sense of the mutualism of interest and sentimental ties" (p. 286). por lo tanto, congruentes con las aspiraciones reales de las personas (p. 12).

Las decisiones que vienen desde arriba y del gobierno se vuelven muy generalizadas y, en el peor de los casos, cumplen las necesidades de un pequeño grupo en la cima de la jerarquía, pues "estas democracias excluyeron de la vida política a las masas populares, privándolos de canales de participación social o de presión política" (p. 12). Peor aún, cuando esas decisiones son de parte de un imperio que oprime un pueblo colonizado, la participación no es considerada. Memmi (1996) lo dice en las siguientes palabras:

La carencia más grande que experimenta el colonizado la constituye el hallarse situado fuera de la historia y fuera de la ciudad. La colonización le suprime toda participación libre así en la guerra como en la paz, toda decisión que contribuya al destino del mundo y al propio, toda responsabilidad histórica y social. (p. 102).

Además, no están fundamentadas las necesidades de un pueblo solo en el individualismo que, como menciona el sociólogo Mayerfeld (2012), no nos prepara mentalmente para reconocer la interconexión que tenemos con nuestro entorno social y ambiental.

Entonces, el desarrollo se aplicaría a lo existente y basado en las necesidades humanas de las comunidades y colectivos, en contraposición a ese individualismo. La constitución social de las comunidades son parte del problema, pero su reconstitución social puede ser parte de las soluciones, esta última depende tanto de las construcciones de nuestra sociedad como de nuestras limitaciones y posibilidades (Bell, 2012). En esta propuesta se reconoce el valor y poder que tienen las comunidades en crear y hacer lo que, de manera colectiva, es beneficioso a la mayoría.

En Puerto Rico hay demasiadas estructuras abandonadas $\mathrm{y}$, antes de utilizar cualquier material, es importante darle el crédito a la conservación arquitectónica como protagonista en el debate del edificio más sostenible, pues implica reusar estructuras que pueden ser recuperadas y minimiza la 
extracción de material crudo. Desde la perspectiva de las ciencias ambientales sería irresponsable recomendar la construcción con tierra para hacer una nueva urbanización o un edificio, en una isla de 111 x 39 millas cuadradas. Tampoco debe construirse en pilotes, como tanto se acostumbra en la isla, por el efecto de latigazo o péndulo invertido que produce en las estructuras un terremoto, ni para edificios altos. Sin embargo, hay remodelaciones, extensiones, pequeñas intervenciones, casas mínimas o alguna estructura nueva que se justifique de manera ambiental, además de que es una alternativa para proveer vivienda a las futuras generaciones.

La propuesta para Puerto Rico es comenzar a adquirir el conocimiento de lo que es la construcción con tierra ya que cobra relevancia cuando entendemos lo que amerita el "principio de precaución", que implica la protección ante la sospecha de un peligro:

Los ejes del nuevo modelo productivo deberán basarse en la utilización de fuentes de energía renovable, en el cierre de los ciclos de materiales, en el mantenimiento de la biodiversidad, en la creación de canales cortos de comercialización y relocalización de la producción y la aplicación del principio de precaución (Herrero, 2016, p. 27).

Y cuando pensamos en la construcción con tierra sabemos que la misma es capaz de hacerse con menos tecnología y que es un material que puede ser reincorporado a la tierra (cierre de ciclos), menor huella ecológica (canales cortos) y definitivamente es una opción al principio de precaución pues es una manera de ver más allá de la condición actual de Puerto Rico como isla y colonia de los Estados Unidos.

Además, existe incertidumbre sobre lo que pueda pasar luego del próximo terremoto y sobre la fecha de caducidad que puedan tener, al menos las estructuras más antiguas en cemento o las que no están bien construidas. Hay una carencia de conocimiento que nos "empodere" sobre cómo hacer autoconstrucción con materiales accesibles que no dependan de la importación y la vulnerabilidad que esto representa como isla y como colonia. Ya estamos experimentando los efectos del cambio climático y seguimos con la dependencia del petróleo y materiales que implican una alta demanda de energía.

La propuesta de comenzar a adquirir el conocimiento de lo que es la construcción con tierra cobra importancia porque es lo que le podemos dejar a generaciones futuras, es sostenible, y una forma de empezar, como propone Daniel Christian Wahl (2017). Para él, la cultura regenerativa es saludable, resiliente y adaptable, se preocupa por el planeta y la vida sabiendo que es la forma más efectiva para crear un futuro próspero para la humanidad. Del mismo modo, cualquier construcción nueva debería tener en consideración su ubicación dentro de las regiones fisiográficas de la isla pues lugares como la costa, los humedales y llanuras aluviales, entre otros, no son los más idóneos para este tipo de construcción, es importante retomar el conocimiento de otros materiales de menor impacto y huella ecológica.

La construcción con tierra es herencia del patrimonio histórico, nos reconecta con nuestros ancestros, con nuestra infancia y con la atracción que sentimos por la tierra, que nos es arrebatada por ideologías impuestas. Bell expone que el individualismo nos alienta a ver nuestros cuerpos separados de los demás y del mundo natural y que las consecuencias de esto es lo que hace que veamos ese mundo natural como sucio, repulsivo y hasta aterrador (Mayerfeld, 2012).

Nos hemos desconectado de nuestra esencia y creamos burbujas asépticas negando nuestras raíces. Para Mayerfeld casi todas las élites adoptan estilos de vida refinados alejados de las consecuencias sudorosas y malolientes de ser animales humanos. En sus propias palabras: "Nearly all elites adopt refined lifestyles that insulate them from the dirty, sweaty, smelly consequences of being a human animal" (p. 161). Sin embargo, desconectarnos de nuestra naturaleza nos ha llevado a darnos cuenta de que somos parte del todo porque de alguna manera buscamos reconectarnos a la fuente y encontrar paz en el bosque, en la tierra 
y en el silencio. La arquitectura con tierra es un conocimiento que empodera y que nos provee las herramientas, conocimientos y materiales para poder edificar con un método de construcción resiliente y saludable para el futuro.

\section{Conclusiones}

A pesar de que los materiales de construcción utilizados en Puerto Rico no son costo-efectivos, ecoeficientes ni sostenibles, son las opciones disponibles y las que conocemos de manera generacional. Cemento, madera y acero poseen virtudes y defectos al igual que la construcción con tierra. Es precisamente el saber lo que nos lleva a forjar una construcción eficiente. La información del comportamiento de un material nos lleva a conclusiones sobre cómo debe ser ese tipo de construcción a nivel estructural, en términos de biohabitabilidad, y cuñales serán los lugares idóneos para construir con él; qué aplicaciones especiales debemos tomar en consideración respecto a nuestro clima particular y cuál es la escala a la que debemos utilizar dicho material. La virtud de las paredes de tierra para regular la temperatura, prometen hacer las paces con la arquitectura tropical y bioclimática que debemos rescatar por ser partícipes y experimentadores del cambio climático. Como consumidores responsables, es importante conocer el impacto del ciclo de vida de cualquier producto y recurrir a un sistema regenerativo y sostenible. Disminuir el uso del cemento es una responsabilidad que debemos asumir. Tanto el material como la vivienda deben ser accesibles al colectivo.

La evidencia demuestra que existieron construcciones con tierra en Puerto Rico, no obstante la falta de continuidad y de transmisión de conocimiento. Aunque existe preocupación sobre el uso de la tierra como material de construcción por la frecuencia de huracanes, susceptibilidad de terremotos/maremotos y el alto índice de precipitación, existe un sinnúmero de investigaciones científicas que sustentan la idea de hacer posible la construcción con tierra mencionada en este escrito. Esto invita a abrir la investigación y conocer sobre prece- dentes en el Caribe e islas vecinas que comparten condiciones similares. Una segunda parte de esta tesis llevará a cabo una serie de pruebas con dos tipos diferentes de suelos de Puerto Rico, esto para evaluar sus propiedades (textura, plasticidad y compactibilidad), transferencia de calor, vapor de agua (absorción y desorción), y erosividad, en bloques de adobe, bloques de tierra comprimida y bloques de cemento. Dichos resultados abrirán la puerta a futuras preguntas y posibilidades.

\section{Referencias bibliográficas}

Abruña, F. (2010). Una casa de madera en el campo. Revista Ambiental Corriente Verde. Recuperado de: [http://www.corrienteverde.com/articulos/ casamaderacampo.html].

ASTM (2010). Standard Guide for Design of Earthen Wall BuildingSystems. PA United States: ASTM International.

Bell, M. M. (2012). An Invitation to Environmental Sociology. California: SAGE Publications, Inc.

Carazas, W. (2017). Test Carazas: Manual pedagógico, Ensayos de correlación de las tres fases de la materia tierra. Guadalajara: Diseños Guevara.

De Hostos, A. (1938). Investigaciones Históricas. San Juan, Puerto Rico: Oficina del Historiados en Colección Puertorriqueña de UPRRP.

Del Cueto, B. (2013). Historia en concreto: el desarrollo de los morteros hidráulicos y el uso del cemento en Puerto Rico. Entorno., 1(22),pp. 12-16.

Del Cueto, B. (2014). La madre naturaleza versus la construcción antillana. Construcción/Arquitectura, pp. 16-17.

Gupta, A. (2011). Tropical Geomorohology. New York, United State: Cambridge University Press.

Herrero, Y. (2016). Una mirada para cambiar la película: Ecología, ecofeminismo y sostenibilidad. Madrid, España: Ediciones Dyskolo.

Herrero, Y. (2016). Una mirada para cambiar la película: Ecología, ecofeminismo y sostenibilidad. Madrid: Ediciones Dyskolo. 
Houben, H. \& Guillaud, H. (2014). Earth Construction: A comprenhensive guide. France: Practical Action Publishing.

Klein, N. (2017). La doctrina del shock: El auge del capitalismo del desastre. España: Liberdúplex, S.L.

Latouche, S. (Noviembre de 2003). Le Monde Diplomatique. Obtenido de quiendebeaquien.org: Recuperado de http://quiendebeaquien.org

Mayerfeld, M. (2012). An Invitation to Environmental Sociology. California, United State: SAGE Publications, Inc.

Max-Neef, M., Elizalde, A. y y Hopenhayn, M. (2010). Desarrollo a escala humana: Opciones para el futuro. Madrid: Biblioteca $\mathrm{CF}+\mathrm{S}$.

Memmi, A. (1969). Retrato del Colonizado. Buenos Aires, Argentina: Ediciones de la Flor.

Metro Puerto Rico (4 de septiembre de 2017). Tormentas y huracanes que pasaron por Puerto Rico. Metro.pr Noticias. Recuperado de: [https://www. metro.pr/pr/noticias/2017/09/04/tormentashuracanes-pasaron-puerto-rico.html].

Muñoz, M. A., Lugo, W. I., Santiago, C., Matos M., Rios, S. \& Lugo J. (2016). Updated Taxonomic Classification of the Soils of Puerto Rico, 2017. Mayaguez, Puerto Rico: University of Puerto Rico- Mayaguez Campus, College of Agricultural Science \& Agricultural Experiment Station and USDANatural Resourses Conservation Service.

Onyegiri, I. \& Ugochukwu I. B. (2016). Traditional Building Materials as a Sustainable Resource and Material for Low Cost Housing in Nigeria: Advantages, Challenges and the Way Forward. Int'l Journal of Research in Chemical, Matallurgical and Civil Engg., 3(2), pp. 247-252.

Pacheco-Torgal, F. \& Jalali, S. (2011). Earth construction: Lessons from the past for future eco-efficient construction. Construction and Building Materials., 29(2012), pp. 512-519 Disponible en: [doi 10.1016/j.conbuildmat.2011.10.054].
Pantel, A. G., Badillo, S. B., Sepúlveda A. \& Del Cueto, B. (1988). Archeological, Architectural and Historical Investigations of the First Spanish Settlement in Puerto Rico. San Juan: The Foundation of Archeology. San Juan: The Foundation of Archaeology, Antropology and History of Puerto Rico.

Rempel, A. R. \& Rempel, A.W. (2016). Intrinsic Evaporative Cooling by Hygroscopic Earth Materials. Geosciences, 6(38), pp. 1-38.

Rivera-Fontán, J. A., Rodriguez-López, J. A. y Rivera-Groennou, J. M. (2011). Investigaciones Arqueológicas en Casa Blanca y sus entornos. 9 no encuentro de investigadores de arqueología $y$ etnohistoria. Instituto de Cultura Puertorriqueña., pp. 89-106.

Red Sísmica (2019). Información sísmica. Terremoto de 1918. Online Puerto Rico Red sísmica, Universidad de Puerto Rico. Recuperado de: [http://redsismica.uprm.edu/Spanish/informacion/terr1918.php].

Suryandari, P. \& Mubin, S. (2007). Mix and Match Traditional and modern technology, toward Sustainable Architecture Housing in the Tropics. 6th Euroseas Conference, ResearchGate, pp. 1-12.

Ecoexploratorio (2018). Terremotos en Puerto Rico. Ecoexploratorio: Museo de Ciencia de Puerto Rico. Recuperado de: [https://ecoexploratorio.org/amenazas-naturales/terremotos/ terremotos-en-puerto-rico/].

Trainer, T. (2013). Some inconvenient theses. Energy Policy, 64(2014), pp. 168-174.

Vicioso, E. (13 de septiembre de 2015). Research Gate. Recuperado de: [https://www.researchgate.net].

Wahl, D. C. (2017). Insurge Intelligence. En: Sustainability is not enough: we need regene-rative cultures.: Recuperado de: [https://medium. com/@designforsustainability/sustainabilityis-not-enough-we-need-regenerative-cultures-

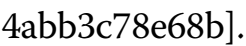

